\title{
Cooperación e innovación en empresas de economía social
}

\author{
Cooperation and innovation \\ in social economy companies
}

Antonio Juan Briones Peñalver'
Juan Gabriel Cegarra Navarro²
Carlos Isidoro López Cano Vieira ${ }^{3}$

Fecha de recepción: 20 de enero del 2012

Fecha de aprobación: 19 de marzo del 2012

Briones, A; Cegarra, J; López, C. Cooperación e innovación en empresas de economía social.

Tecnología en Marcha. Vol. 25, Nº 6. Pág I2-22

I Profesor, Universidad Politécnica de Cartagena, Departamento de Economía de Empresa, Facultad de Ciencias de la Empresa, España. Teléfono: (34) 968325943.

Correo electrónico: aj.briones@upct.es

2 Profesor, Universidad Politécnica de Cartagena, Departamento de Economía de Empresa, Facultad de Ciencias de la Empresa, España. Teléfono: (34) 968325788. Correo electrónico: juan.cegarra@upct.es,

3 Profesor, Universidad Politécnica de Cartagena, Departamento de Economía de Empresa, Facultad de Ciencias de la Empresa, España.Teléfono: (351) 969737।43.

Correo electrónico: carlos.cano@clix.pt 


\section{Palabras clave}

Cooperación, alianzas, innovación, sistema.

\section{Resumen}

Las empresas, al formar acuerdos de cooperación, desarrollan medidas de innovación y nuevas formas organizacionales. Mediante las alianzas con terceros (UTEs, joint-ventures, acuerdos con proveedores, acuerdos comerciales) pueden articular mecanismos propulsores de la innovación, que a su vez son críticos para competir con las empresas de economía social (EES). El objetivo de este estudio es entender las capacidades generadas por la innovación, las cuales utilizan procesos colaborativos. El marco empírico del trabajo tiene como finalidad estudiar los procesos de cooperación llevados por las EES de la Región de Murcia, con el propósito de conocer sus argumentos en la formación de acuerdos, identificar las variables que afectan a su desarrollo y, conocer los factores determinantes que impulsan a las EES a la dinamización de procesos de cooperación e innovación.

\section{Key words}

Cooperation, alliances, innovation, system.

\section{La cooperación interempresarial}

El concepto de cooperación interempresarial se entiende como un acuerdo o alianza entre organizaciones, en el que cada una conserva su independencia jurídica, y no existe relación de subordinación entre ellas. Las organizaciones coordinan el desarrollo de actividades conjuntas, así como el uso de recursos, con el propósito de alcanzar uno o varios objetivos previamente definidos y considerados relevantes para la satisfacción de las necesidades o fines propios de cada una de ellas. La cooperación interempresarial constituye una valiosa herramienta para atender adecuadamente a aquellas necesidades o problemas de las organizaciones que por sí solas difícilmente podrían atender o lo harían en peores condiciones.

De la variedad de mecanismos existentes, se toma en cuenta la siguiente clasificación: (I) acuerdos

\begin{abstract}
The companies when forming cooperation agreements develop innovation measures and new organizational forms. Through the alliances with a third partner (UT, joint-ventures, agreements with suppliers, commercial agreements) mechanisms propellers of the innovation can be articulated in order to be more critic to compete the companies of social economy (EES). The objective of this study is to understand the capacities generated by the innovation used for collaborative processes. The empiric mark of the work has as purpose to study the cooperation processes taken by the EES of the Region of Murcia, with the purpose of knowing its arguments in the formation of agreements, to identify the variables that affect to its development, and to know the decisive factors that impel the EES, to the revitalization of cooperation processes and innovation.
\end{abstract}

contractuales, (2) acuerdos accionariales, (4) redes empresariales y (5) acuerdos informales.

Los acuerdos contractuales permiten la utilización conjunta de recursos sin que por ello dé lugar a la creación de una nueva entidad con personalidad jurídica propia. Entre los más importantes destacan:

I. Los contratos de larga duración sobre actividades concretas, los cuales dan lugar a una relación continuada entre las partes cooperantes.

2. Las franquicias, que son un tipo de contrato entre empresas, mediante el cual una empresa -el franquiciador-cede a otra u otras - franquiciado o franquiciados el derecho a la comercialización de determinados productos o servicios a cambio de determinadas compensaciones económicas directas o indirectas.,

3. La licencia o derechos de propiedad industrial, este tipo de acuerdo es un contrato por el 
que una empresa -la licenciante- otorga a otra -la licenciataria- el derecho a utilizar sus derechos de propiedad industrial mediante una contraprestación.

4. La subcontratación consiste en que una empresa, la principal, encarga a otras empresas -las subcontratadas- la producción de una parte más o menos importante de los componentes de sus productos.

5. Los acuerdos spin-off desde una empresa fomentan o apoyan financiera y económicamente a sus trabajadores cualificados propios para que abandonen sus puestos de trabajos y creen su propia empresa.

6. Los consorcios son acuerdos de colaboración realizados con un horizonte temporal cierto y tienen como objeto el desarrollo o ejecución de una obra, servicio o suministro concreto de manera conjunta.

Los acuerdos accionariales son aquellos los cuales se incluyen acuerdos de capital. Se distinguen dos categorías. La primera, joint venture o empresa conjunta, es una asociación por la que dos o más empresas crean una entidad para que lleve a cabo una actividad económica productiva. Los rasgos más característicos de este tipo de acuerdo son: la existencia de una comunidad de intereses, la realización de negocios en común, la aportación conjunta de recursos, compartir riesgos y disfrutar de los beneficios o pérdidas, así como prolongar la relación de colaboración. El segundo tipo de acuerdos accionariales son los de participación minoritaria, en el cual la empresa participa en el capital social de otra como accionista, lo que supone un vínculo entre dos o más empresas.

Las redes empresariales son una forma organizativa intermedia entre el mercado y la empresa caracterizada por la pluralidad de acuerdos de cooperación entre los diferentes colectivos o grupos de interés (proveedores, clientes, competidores, instituciones públicas y privadas, etc.). Destacan entre otras formas de redes empresariales: (1) los acuerdos - alianzas con empresas de la industria, (2) con variedad de empresas participantes, (3) y con proveedores, distribuidores y clientes, en función de los objetivos que se persigan y de los colectivos que estén dispuestos a cooperar para conseguirlos. Finalmente, también forma parte de la clasificación los acuerdos informales entre los distintos grupos de interés empresariales.

Propuestas para la formación de acuerdos de cooperación

La realidad empresarial en torno al desarrollo de Redes Inter organizativas de Empresas e Instituciones y la formación de alianzas donde participan las instituciones en aspectos referidos a la innovación, la investigación y el desarrollo llevan a plantear la estrategia de cooperación como opción que contribuye a mejorar la ventaja competitiva de las empresas.

Las condiciones de subcontratación y la colaboración con los grupos de interés son determinantes para la formación de acuerdos de cooperación (Zhao et al, 2005; Narayanan y Fahey, 2005; Li, 2005; Harris y Wheeler, 2005; Singh y Mitchell, 2005; Fogarty y Rogers, 2005; Doh, 2005). La cooperación es un proceso dinámico (Malhotra et al., 2005) y las empresas generan sinergias recíprocas al colaborar estrechamente y ejecutar las tareas mediante un proceso iterativo en el que comparten sus conocimientos (Dyer et al., 2005).

\section{Cooperar para innovar}

La innovación es un avance aplicado al desarrollo tecnológico que involucra a un nuevo producto, un nuevo servicio, nuevas prácticas, procesos o nuevas tecnologías (Schumpeter, 1939), así como la contribución de otras fuentes de conocimiento.

En la actualidad, la innovación puede tener acepciones distintas (Silva, 2004). Así, la innovación tecnológica definida como comprometer determinados recursos para el desarrollo de nuevos productos o la mejora de procesos, en los sistemas organizativos y cambios en la estructura de propiedad. Además, si procede, se puede intervenir la actividad inventiva con el fin de posibilitar la creación y mejora de bienes y servicios.

La introducción de una nueva tecnología después de su invención se refleja en el uso y creación de conocimiento necesario para competir o definir un liderazgo (Tidd, 1993; Jassó, 2004). En este sentido, el mercado actúa como un agente regulador e impulsor de innovaciones cuyo objetivo puede ser incrementar la competitividad. Pero requiere un conjunto diferente de conocimientos y destrezas dentro del marco de una estrategia corporativa racionalista que 
permita implementar la estrategia innovativa, según lo señalan Tidd, Bessant y Pavitt (1997).

Por otro lado, desde esta estrategia innovativa, Teece y Pisano (1994) desarrollaron el enfoque de las capacidades dinámicas con el objetivo de explotar las capacidades o aptitudes distintivas. Para lograrlo, la empresa analiza el ambiente externo en búsqueda de oportunidades, es decir, de innovaciones que le otorguen ventajas competitivas (Malaver y Vargas, 2005). Esto origina una reflexión estratégica que puede dar lugar a innovaciones (Tidd, 2000) enfocada a favor de la colaboración y los modelos de cooperación entre firmas. Fernández (2006) afirma que esta búsqueda de la innovación de las organizaciones se da por medio de las alianzas y la colaboración con terceros (UTEs, joint-ventures, acuerdos con proveedores, acuerdos comerciales, etc.), quienes pueden articular mecanismos propulsores de la innovación y, por tanto, fundamentales o críticos para competir.

Adams, Bessant y Phelps (2006) afirman la existencia de un gran deseo en el mundo académico para que se encuentren los conceptos, las diferentes terminologías y la acumulación de conocimientos en todos los aspectos referidos a la innovación empresarial. Por ello, esas discordancias antes aludidas crean confusión en torno al concepto, otras fuentes de conocimiento y las diferencias de competitividad entre sectores industriales (Hernández y De la Calle, 2004).

En este artículo, se entiende la innovación como una estrategia empresarial, fruto de la colaboración entre firmas y resultado de las relaciones inter organizativas, es decir, de la cooperación interempresarial, particularmente aquellas que establecen las empresas entre sí y además en colaboración con la administración pública (instituciones, universidades, centros de investigación), lo cual les permite trabajar en un nuevo entorno global empresarial. El estudio de los procesos de innovación en este trabajo tiene como propósito, el diseño y la evaluación por parte de los entes públicos de políticas destinadas a fortalecer los sistemas de innovación.

\section{Sistemas de innovación}

El concepto de sistemas de innovación fue recogido originalmente por Lundvall (1985), como las relaciones entre firmas e sus interacciones con centros tecnológicos (Lundvall, 1999). Estos sistemas vinculan la innovación con las Pymes mediante la articulación de los proveedores, las tecnologías, la formación, el aprendizaje y la confianza para cambiar con afán de crecimiento. De acuerdo con Goñi (2006), estas son las variables más significativas de la innovación, y permiten una actuación concreta y sistemática de los programas públicos

Entender la importancia de las capacidades generadas por la innovación y el uso del conocimiento lleva a las autoridades públicas a realizar continuados esfuerzos para la investigación en la transferencia de tecnología de los sistemas de innovación (Arnold y Thuriaux, 1997). Además, según Arnold y Kuhlman (200l) de los fallos de mercado, existen otros cuatro que requieren intervención política para mejorar los sistemas de innovación: la incapacidad para la innovación, los fallos institucionales, los fallos en las redes de los actores y los fallos estructurales (Arnold y Kuhlman, 200 I).

En este sentido, las medidas de cooperación y colaboración en las pymes se consideran una herramienta de importancia estratégica para el crecimiento junto con las actividades de innovación. Así mismo, pueden guiar las acciones públicas y privadas tendientes a mejorar el desempeño de las empresas en los mercados e impulsar el desarrollo económico y social.

Entre las propuestas con que cuentan las empresas para encarar procesos de innovación se puede sugerir: (I) ampliar la formación o nivel de estudios de los empleados; (2) adoptar mecanismos o sistemas de control de calidad y el empleo de herramientas estadísticas; (3) averiguar y adoptar lo actuado por las empresas en relación a la incorporación de nuevas Tecnologías de Información y Comunicaciones (TICs) y (4) ampliar las consultas con respecto a la existencia de relaciones interorganizativas con los restantes agentes del sistema de innovación no circunscritas en acuerdos formales de cooperación, sino que abarquen las múltiples y variadas posibilidades de interacción entre los agentes.

El análisis de los tipos de sistemas de innovación regional o local lleva a estudiar diferentes contextos de conocimiento a partir de las diferentes industrias económicas (Asheim, 2005) y del papel de las instituciones en la interacción del conocimiento entre los agentes (Lundvall, 1998). Cooke (200I) considera que existen diferentes condicionantes (geográficos, económicos, sociales) que definen 
diferentes posturas científicas y comportamientos para un estudio detallado.

Un sistema regional o local de innovación debe servir para analizar la adaptación de los territorios a las nuevas situaciones tecnológicas e industriales. Debe ser un instrumento para planificar, dinamizar y hacer competitivos los territorios, mediante la interacción, movilización y regulación de agentes, recursos e infraestructuras. Las actividades de investigación de los sistemas de innovación se han convertido en un factor crítico para la competitividad de las regiones y de las ciudades, lo cual ha permitido el surgimiento de nuevas líneas de investigación sobre estructuras productivas, desarrollo organizativo y medio ambiental que aspiran no solo a mejorar el entorno, sino a elevar la calidad de vida de los ciudadanos (Tidd, 200 I; Tidd y Bodley, 2002; Bessant, 2005; Asheim, 2005, entre otros).

Aún existen vacíos en indicadores globales para tipificar el comportamiento de las empresas en innovación, por lo que se recomienda su estudio a partir de la colaboración con el entorno científicotécnico y empresarial March (2003). Por tanto, la importancia de optimizar la gestión de la información dentro de los sistemas de innovación empresarial viene dada por la necesidad de lograr un mejor posicionamiento en el entorno globalizado, con un proceso de toma de decisiones con menor incertidumbre, ante escenarios de enorme complejidad y diversidad.

\section{Marco empírico}

Población y recolección de datos

Para el desarrollo del trabajo empírico se han realizado encuestas a firmas de economía social, todas ellas situadas en el término municipal de Murcia (España). Dichas firmas están asociadas a la Unión de Cooperativas de Trabajo Asociado de la Región de Murcia (UCOMUR) y a la Asociación de Empresas de Economía Social de la Región de Murcia (AMUSAL). La población objeto de estudio representa empresas de varios sectores, entre los cuales, los más representativos son el sector secundario (industria y construcción) y el sector terciario (servicios y mantenimiento). Se obtuvieron 70 cuestionarios completos, de los cuales 36 fueron contestados por cooperativas y 34 por sociedades laborales.
Estas firmas son consideradas pequeñas unidades de producción, comercio o prestación de servicios, en las que se pueden distinguir elementos de capital, trabajo y tecnología. Los responsables, claramente emprendedores y forzados por las circunstancias, desarrollan variadas actividades de producción y servicios.

\section{Análisis estadístico y tratamiento de datos}

Las variables se midieron con escalas Likert de cinco puntos - donde un valor de I significa un desacuerdo total del individuo con el ítem, y un valor de 5 significa un acuerdo total con el ítem-, en las cuales se solicitó a la persona encuestada que expresara su grado de acuerdo con el ítem. Previo al tratamiento de los datos, se llevó a cabo un análisis descriptivo exploratorio, con el objeto de conocer para cada una de las variables la media, la desviación típica, la mediana, la moda, y así poder determinar pautas de comportamiento de las empresas.

Ante la presencia de no normalidad en la muestra, se realizó el análisis de fiabilidad para el cual los valores recomendados son para Alfa de Cronbach, comprendidos en el intervalo $(0,7-0,9)$.

Se realizarán análisis factoriales utilizando como método de extracción de factores el de Componentes Principales (ACP), pues maximiza la varianza total explicada con el mínimo número de factores extraídos. La rotación de los factores empleados es "Varimáx", por ser una rotación ortogonal que minimiza el número de variables con saturación máxima en cada factor.

El análisis factorial consta de cuatro fases características: I) el cálculo de una matriz capaz de expresar la variabilidad conjunta de todas las variables; 2) la extracción del número óptimo de factores; 3) la rotación de la solución para facilitar su interpretación y 4) la estimación de las puntuaciones de los sujetos en las nuevas dimensiones.

En relación con las cargas factoriales de los ítemes, fueron elegidos aquellos con al menos una carga del 0,50 (Hair et al., 200 I), se aplicó el test de Kaiser Meyer Olkin (KMO) para explicar el grado de adecuación muestral y se realizó la prueba de esfericidad de Barlett para estudiar los valores resultantes.

El test de Kaiser Meyer Olkin (KMO) mide el grado de adecuación muestral, compara los coeficientes de correlación observados con los coeficientes de 
correlación parcial, los cuales indican la fuerza de las relaciones entre dos variables y a la vez eliminan la influencia de otras variables. Si los valores obtenidos alcanzan niveles superiores a 0.5 , este índice se pueden aceptar, teniendo la consideración de que valores superiores del índice permiten obtener a mejores intervalos de aceptaciónı.

Con el contraste de esfericidad propuesto por Barlett2 se pretende dar respuesta a si las variables dependientes del modelo están correlacionadas, es decir, se trata de determinar si existe o no una relación significativa. Este contraste indicaría una posible existencia de factores subyacentes en la escala, lo que justifica la conveniencia de la aplicación de esta técnica. Los valores pueden fluctuar entre 0 y I, cuando son muy próximos a 0 se consideran aceptables, lo que implica que existe una única variable latente $y$, por tanto, se mantienen todos los ítemes que la conforman.

Si los parámetros relativos al KMO y la prueba de esfericidad de Barlett son aceptables, se considera que las variables se pueden recoger en un solo factor; así, se puede examinar las subsiguientes apreciaciones sobre la obtención de más factores. Para ello, observamos los índices de Comunalidades (Comun.) o la proporción de las variables explicables por el modelo factorial. Si estas son altas, entonces la proporción de la varianza explicada por el modelo factorial es buena.

Se utilizará el modelo de regresión logística, pues permite predecir la proporción de una de las categorías de la variable dependiente, en función de una o más variables independientes. En el modelo de regresión logística binaria (RLB), el carácter no métrico de la variable dependiente dicotómica, se adecúa haciendo predicciones de pertenencia a un grupo y la probabilidad de ocurrencia de un suceso (Hair et al., 200 I). Con este modelo lo que interesa es saber cómo afectan los cambios en los elementos a las probabilidades de respuesta.

La idoneidad predictiva está en función de I) la interpretación sustantiva, donde las variables independientes que intervienen están en función de las hipótesis de investigación además de que la interpretación de esas relaciones debe ser teóricamente

I Intervalo de aceptación muy bueno (0,8-1).

2 Por la prueba de esfericidad de Bartlett ( $\mathrm{p}$-valor $<0$ '05), entonces la técnica análisis factorial es adecuada para nuestros datos. relevante; 2) su capacidad de predicción estadística, pues debe ser significativa y 3 ) debe cumplir el principio de parsimonia, es decir, que con un menor número de variables presente mejor bondad de ajuste.

La regresión logística utiliza el método de máxima verosimilitud3, de modo que los coeficientes que estima el modelo hacen los datos "más verosímiles" (Visauta y Martori, 2003). Además, pese a que el modelo estimado pueda ser adecuado y útil, los estadísticos que indican las medidas de bondad del ajuste, son únicamente orientativos; pudiendo adoptar valores moderados o incluso bajos, (Pardo y Ruiz, 2002). En este sentido, el estadístico de Wald contrasta la hipótesis de si los coeficientes de regresión son iguales a cero.

El criterio de significación estadística que se suele adoptar es: $\alpha=0.05$. Si el coeficiente de Wald arroja valores significativos ( $p$-valor $<0.05$ ), entonces se puede afirmar que las variables independientes (explicativas) contribuyen significativamente a explicar el comportamiento de la variable dependiente (o criterio).

Las pruebas de pseudos $-R^{2}$ y de $R_{L}^{2}$ obtienen valores entre 0 y $\mathrm{I}$, de manera que cuanto más se acerquen al valor I, mejor será la bondad del ajuste. Las medidas chi-cuadrado de Pearson o de Hosmer y Lemeshow en combinación con el coeficiente de Wald, suministran apoyo para aceptar que el modelo de regresión logística es significativo y adecuado para un análisis posterior4.

Los valores bajos de la prueba de chi-cuadrado de Pearson los cuales están asociados a valores de significación estadística elevados, son indicadores de que el modelo se ajusta bien a los datos, por tanto, un valor de la chi-cuadrado de Pearson no significativo ( $p$-valor>0.05) muestra una adecuada bondad de ajuste. En el caso de que el modelo incluya una o más variables independientes de tipo cualitativo, se recomienda la prueba chi-cuadrado de Hosmer y Lemeshow la cual mide la correspondencia de los valores reales y predichos de la variable dependiente (criterio), procediendo igual que con el contraste anterior, y si el p-valor $>0.05$, entonces se acepta el modelo seleccionado a los datos.

3 La razón de verosimilitud sigue una distribución chi-cuadrado (Bisquerra-Alzina, 1989).

$4 \quad$ Para su interpretación se siguen los manuales de: Bizquerra-Alzina (1989); Hair et al. (200 I); Pardo y Ruiz (2002, 2005); Visauta y Martori (2003) y Pérez-López $(2004,2005)$. 
Una vez realizado el contraste de hipótesis, es importante conocer la interpretación de los coeficientes de regresión de las variables incluidas en la ecuación, para lo cual se deben interpretar las variables independientes significativas. Un coeficiente positivo aumenta la probabilidad de pertenencia al grupo, interpretándose como un incremento en la probabilidad que el individuo tome el valor criterio debido a una variación unitaria en la variable independiente. Por su parte, un valor negativo debe explicarse como una disminución en la misma probabilidad. Valores altos de la chi-cuadrada parcial indica que el término en cuestión es importante para el ajuste.

\section{Resultados de la investigación}

En primer lugar, se expone la descripción de las preguntas del cuestionario relativas a las relaciones inter organizativas, las alianzas estratégicas, los acuerdos, y los procesos de cooperación con otras firmas, instituciones, centros de investigación, asociaciones.

En lo relativo al análisis de la experiencia previa en cooperación interempresarial, las respuestas obtenidas indican que la mayoría (80\%) habían mantenido relaciones inter organizativas, además de haber contado con experiencia previa de colaborar con instituciones y centros de investigación para favorecer la innovación tecnológica.
En el gráfico I se muestran los posibles colectivos que se prestan para llevar relaciones inter organizativas. Se observa una mayor predisposición a cooperar con clientes y proveedores, seguida de la experiencia en colaboraciones con las administraciones públicas, las empresas de sectores distintos y las asociaciones representativas.

De acuerdo con la exposición teórica, en el gráfico 2 se presentan las razones de tipo estratégico que llevan a las empresas a cooperar; el "enfoque de recursos y capacidades" es el argumento mejor valorado (ítem con media $=4,29$ ). A continuación, y por orden de consideración media, aparecen los ítemes referentes a la "mejora de la eficacia en los sistemas de trabajo" (ítem con media $=4,21$ ), el "acceso a proyectos de investigación, desarrollo e innovación" (ítem con media = 3,76), "crear y compartir redes comerciales" (ítem con media $=3,70$ ), la "superación de las barreras de los mercados" (ítem con media $=3,65$ ), "creación y explotación de sinergias" ('́tem con media = 3,62), y la "reducción del riesgo y la incertidumbre" (ítem con media $=3,61$ ).

El número de variables que valora las razones por las que decide o decidiría cooperar la empresa ( 9 ítemes) ofrece el valor Alfa de Cronbach $=0,87 \mathrm{I}$. Este valor indica consistencia interna entre los elementos de la escala, ya que es superior a los

Proveedores

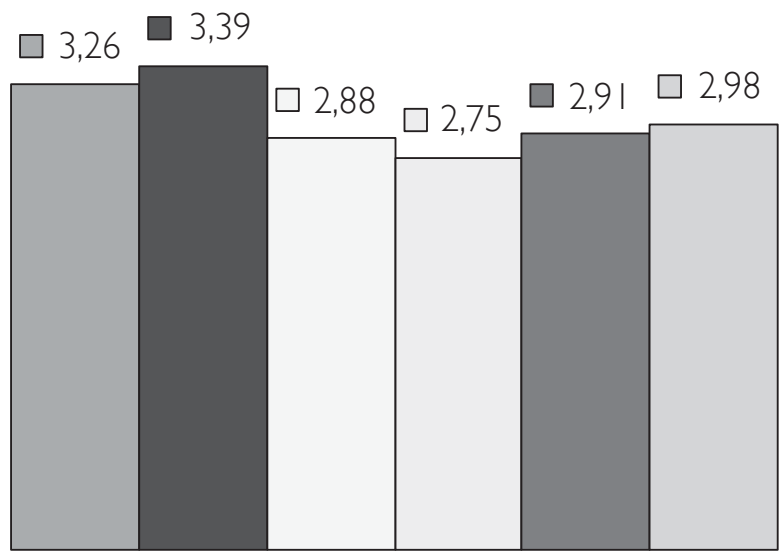

$\square$ Clientes

Competidores

Asociaciones

Empresas de sectores distintos

Administraciones públicas 


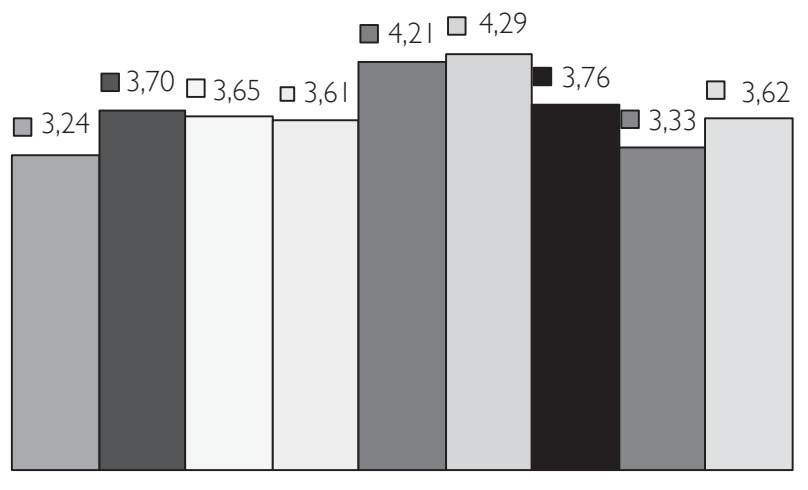

MEDIA
- Especializarse en productos y servicios de la industria

- Crear y compartir redes comerciales

口 Superar las barreras de los mercados.

口 Reducir el riesgo y la incertidumbre

- Mejorar la eficacia en sistemas de trabajo

- Acceder a nuevos recursos y capacidades

Acceder a proyectos de investigación, desarrollo e innovación

- Masa crítica-obtener un tamaño adecuado para competir

Crear y explotar sinergias

Gráfico 2. Razones por las que decide o decidiría cooperar su empresa.

siguientes considerados como referencia $(0,6-0,7)$ y los valores recomendados son para el intervalo $(0,7-0,9)$.

El análisis factorial, como técnica de reducción de la dimensionalidad, denota que las comunalidades informantes de la proporción de la varianza explicada son altas ante la presencia de un único factor. La medida de adecuación muestral Kaiser-Meyer-Olkin (KMO) es de 0,746a. La prueba de esfericidad de Barlett arroja una chi-cuadrado significativa, lo cual indica que las razones por las que decide cooperar la empresa se pueden recoger en un único factor.

En el gráfico 3 se observa que las empresas manifiestan una preferencia por los posibles acuerdos de tipo contractual, entre ellos los "contratos de larga duración sobre actividades concretas", así como la "subcontratación continuada en régimen de cooperación". También es de destacar las preferencias de cooperación a través de las diferentes redes inter organizativas presentadas. Estos resultados indican la preferencia por las redes inter organizativas de tipo vertical, para llevar a cabo acuerdos con los grupos de interés: proveedores, distribuidores y clientes.

Se empleó el modelo de regresión logística, el cual permite predecir la proporción de una de las categorías de la variable dependiente, en función de una o más variables independientes, para hacer predicciones sobre las siguientes apreciaciones:
Proposición) La probabilidad de mantener acuerdos de cooperación en un futuro (variable dependiente, Sí(No), puede depender de las variables explicativas: razones (factor único), los costes de la cooperación y las diferentes ventajas adheridas a los mecanismos de cooperación.

La tabla de clasificación indica que el modelo es bueno para predecir en el futuro, pues si se aplica la clasificación a las observaciones ya conocidas se obtiene un porcentaje de éxitos del 84,8\%. El estadístico de Wald (Sig. = 0.000), que contrasta la hipótesis de si los coeficientes de regresión son iguales a cero, presenta un valor significativo ( $p$-valor $<0.05)$, entonces se puede afirmar que las variables independientes (explicativas) contribuyen significativamente a explicar el comportamiento de la variable dependiente (o criterio).

El $R^{2}$ de Cox (valor $\left.=0.275\right)$, Snell y Nagelkerke (valor $=0.448$ ), la prueba de ajuste de HosmerLemeshow (Sig. $=0.348$ ), con un $p$-valor $>0.05$ indica buen ajuste del modelo a los datos. Las medidas chi-cuadrado de Pearson, Hosmer y Lemeshow no son significativas ( $p$-valor> 0.05). Esto es indicativo de una adecuada bondad del ajuste, entonces se acepta el modelo seleccionado.

La tabla I integra las once variables incluidas en la ecuación, y muestra las estimaciones de los coeficientes del modelo y los datos para valorarlo: 


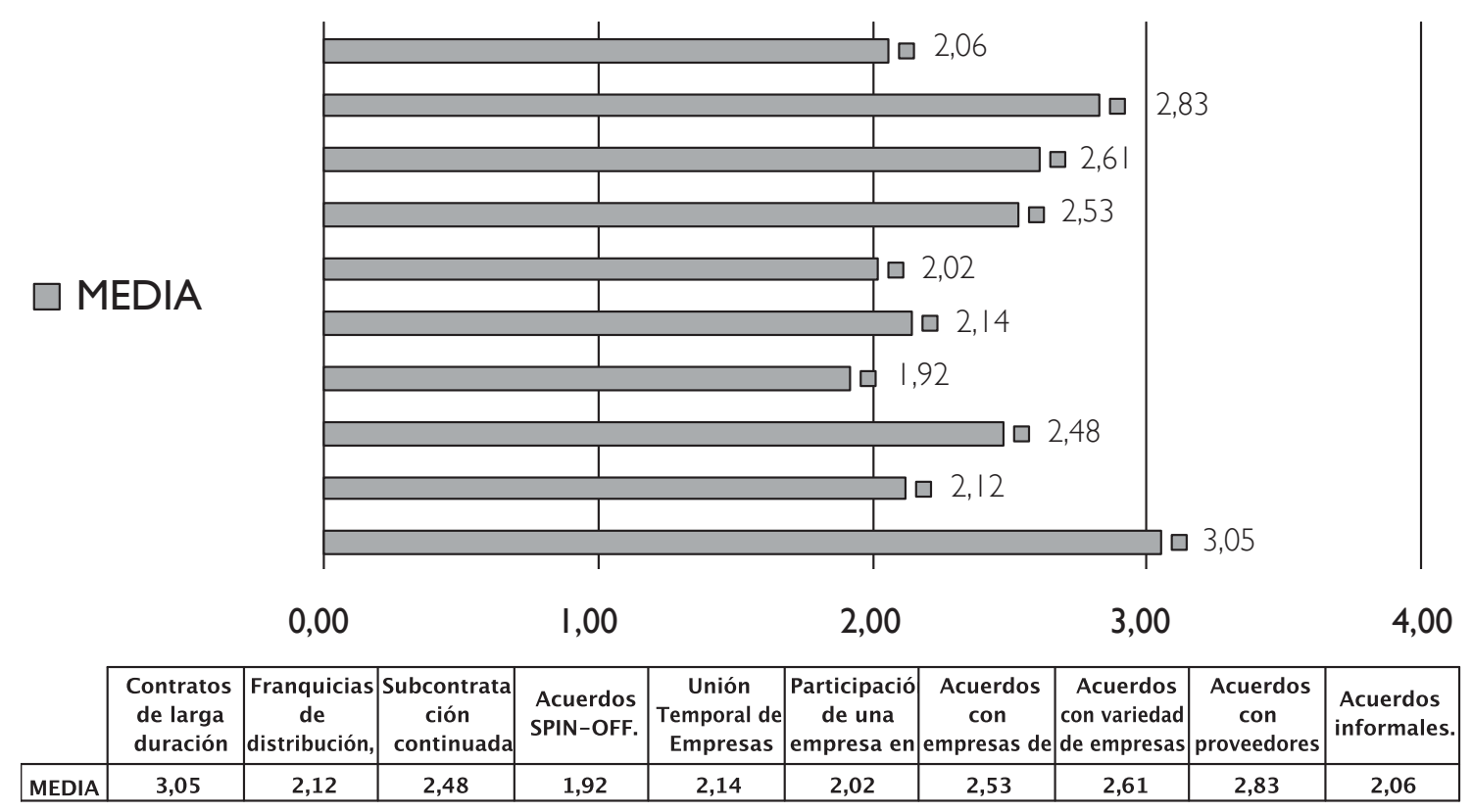

Gráfico 3. Mecanismos de la Cooperación Empresarial.

\begin{tabular}{|l|c|c|c|}
\hline \multicolumn{1}{|c|}{ Resultados modelo } & B & Wald & Exp (B) \\
\hline HLB- I: Razones para llevar acuerdos (factor I) & 0.104 & 0.02 | & I.II0 \\
\hline HLB-2: Costes de la cooperación (factor 2) & 0.589 & 1,179 & 1.802 \\
\hline HLB-3: Especialización en productos y servicios & 0.634 & 1,107 & 1.886 \\
\hline HLB-4: Crear y compartir redes comerciales & -2.69 I & $7,293 a$ & 0.068 \\
\hline HLB-5: Superar las barreras de los mercados & 1.020 & 1.888 & 2.774 \\
\hline HLB-6: Reducir el riesgo y la incertidumbre & 0.642 & 0.977 & 1.900 \\
\hline HLB-7: Mejorar la eficacia y los sistemas de trabajo & -0.725 & 1.118 & 0.484 \\
\hline HLB-8: Acceder a nuevos recursos y capacidades & 0.208 & 0.060 & 1.231 \\
\hline HLB-9: Acceder a proyectos de I+D+i & 0.611 & 1.105 & 1.842 \\
\hline HLB- I0: Obtener un tamaño adecuado para competir & -0.031 & 0.003 & 0.970 \\
\hline HLB-II: Crear y explotar sinergias entre las empresas & -0.229 & 0.260 & 0.795 \\
\hline (Constante) & -0.215 & 0.005 & 0.807 \\
\hline
\end{tabular}

Tabla I: Modelo de regresión logística binaria (RLB).

Fuente: Elaboración propia. Donde: Sig.: $p<0.0$ la; $p<0.05 b, p<0$. Ic.

Una vez realizado el contraste de hipótesis también es importante saber la interpretación de los coeficientes de regresión de las variables incluidas en la ecuación, para lo cual se debe interpretar las variables independientes significativas. Los resultados del análisis de la asociación descrita en el modelo I advierten que la única razón o ventaja esperada de seguir los modelos de cooperación con cierta evidencia empírica para la predicción es la posibilidad de crear y compartir redes comerciales".
Sin embargo, el coeficiente $(B=-2.69$ I) tiene signo negativo, lo cual indica que la probabilidad de mantener acuerdos de cooperación en un futuro es más probable en aquellas empresas de economía social que no comparten redes comerciales. Dado que los coeficientes (B) están expresados en la métrica original, la probabilidad de mantener acuerdos en el futuro se pueden presentar con mayor probabilidad en las empresas que consideran positivamente a la cooperación porque: I) lleva a superar determina- 
das barreras en los mercados; 2) reduce el riesgo y la incertidumbre; 3) lleva a la especialización en productos y servicios y 4) sirve para acceder a proyectos de investigación, desarrollo e innovación $(1+D+i)$.

\section{Conclusiones y líneas de investigación futuras}

El marco teórico proporciona una descripción de las medidas de colaboración entre firmas y los procesos de cooperación diseñados para determinados procesos de innovación en las empresas de economía social. Este trabajo pretende establecer las razones de las EES para llevar a cabo mecanismos de cooperación.

En relación con el análisis empírico en las entidades de la economía social murciana, la mayoría de los encuestados cuenta con experiencia previa en cooperación al haber mantenido relaciones inter organizativas con centros de innovación tecnológica. Además, existe predisposición para cooperar con los diferentes grupos de interés, de manera tal que las colaboraciones con proveedores y clientes son mejor recibidas, es decir, se prefieren los stakeholders que conforman directamente actividades primarias de la cadena de valor de la empresa.

Los motivos de tipo estratégico para llevar a cabo procesos de cooperación, en términos de mayor acceso a recursos y capacidades recoge el mejor valor medio. Quizás por ello, son muy bien considerados aquellos mecanismos administrativos para formalizar acuerdos que suponen contratos de larga duración sobre actividades concretas.

Finalmente, la probabilidad de llevar a cabo nuevos acuerdos de cooperación depende de determinadas variables, teniendo más importancia las que aluden a motivos comerciales, estrategias de especialización y el acceso a sistemas de innovación que posibilitan la participación en proyectos de Investigación, desarrollo e Innovación de la entidad de economía social.

\section{Bibliografía}

Adams, R.; Bessant, J. \& Phelps, R. (2006). Innovation management measurement: a review. En Internacional Journal of Management Reviews, 8( I), pp. 2 I-47.

Arnold, E. \& Kuhlman, S. (200I). RCN in the Norwegian Research and Innovation System, informe de base $N^{\circ} 12$ de la evaluación del Consejo de Investigación de Noruega, Oslo: Ministerio de Ecuación, Investigación y Asuntos Religiosos del Reino de Noruega. Disponible en http://www. technopolis-group.com

Arnold, E., \&Thuriaux, B. (1997). Developing Firms, Technological Capabilities. Informe para la OCDE, Brighton. Disponible en www.technopolis-group.com

Asheim, B. (2005). Knowledge bases and regional innovation systems: comparing nordic clusters. En Research Policy, 34(8), pp. | $173-1190$.

Bessant, J. (2005). Enabling continuous and discontinuous innovation: learning from the private sector. En Public Money \& Management, vol. 25, $\mathrm{N}^{\circ}$ I, pp. 35-43.

Bisquerra-Alzina, R. (1989). Introducción conceptual al análisis multivariable. Barcelona: Promociones y Publicaciones Universitarias, S.A.

Cooke, P. (200I). Regional innovation systems, clusters, and knowledge economy. Centre for Advanced Studies, University of Wales: Oxford University Press.

Doh, J.P. (2005). Offshore outsourcing: implications for international business and strategic management. Theory and practice. Journal of Management Studies, 42(3), mayo, pp. 695-704.

Dyer, J.H.; Kale, P., \& Singh, H. (2005). Cuándo aliarse y cuándo adquirir empresas. En Harvard Deusto Business Review, |31, enero, pp. 7|-79.

Fernández, D. (2006). El director de sistemas como impulsor de la innovación en la empresa. En Universia Business Review, $3^{\circ}$ trimestre, II, pp. 104- | |1.

Fogarty, T.J. \& Rogers, R.K. (2005). Financial analyst's reports: an extended institutional theory evaluation. En Accounting, Organizations and Society, 30(5), mayo, pp. 331-356.

Goñi, J.j. (2006). Herramientas para la innovación regional: el impulso desde la administración a las Pymes. En Tecnimap. Simposio/conferencia llevado/llevada a cabo por Nombre de la Organización, Sevilla.

Hair, J.F. et al. (200I). Análisis multivariante.(6ta Ed.) Madrid: Prentice Hall

Harris, S. \& Wheeler, C. (2005). Entrepreneur's relationships for internationalization: functions, origins and strategies. En International Business Review, 14(2), pp. I87-207.

Hernández, R. \& De La Calle A. (2004). El comportamiento innovador y los resultados de la empresa: un análisis empírico. En XVIII Congreso Anual y XIV Congreso HispanoFrancés de AEDEM, Orense.

Jassó, J. (2004). Trayectoria tecnológica y ciclo de vida de las empresas: una interpretación metodológica acerca del rumbo de la innovación. En Contaduría y Administración septiembre-diciembre, 214. Obtenido desde http://www. contaduriayadministracionunam.com.mxLi, L. (2005). The effects of trust and shared vision on inward knowledge transfer in subsidiaries intra-an inter-organizational relationships. En International Business Review, I4(I), febrero, pp. 77-95. 
Lundvall, B. (1985): Product Innovation and User-Producer Interaction. Aalborg, Denmark: Aalborg University Press.

Lundvall, B. (1998): Why study national systems and national styles of innovation?, Technology Analysis \& Strategic Management, vol. 10 (4), pp. 407.

Lundvall, B. (1999): National business systems and national systems of innovation, International Studies of Management \& Organization, vol. 29 (2), pag. 60-77.

Malaver, F. \& Vargas, M. (2004). Los procesos de innovación en la industria colombiana: resultados de un estudio de casos. En Cuadernos de Administración, 17 (28).

Malhotra, A.; Gosain, S. \& El Sawy, O. (2005). Absorptive capacity configurations in supply chains: gearing for partner-enabled market knowledge creation. En MIS Quarterly, 29(I), pp. |45- 188.

March, I. (2003), La medición del desempeño ante la innovación mediante el uso de indicadores y macroindicadores. En Revista de Investigación en Gestión de la Innovación y Tecnología, 20, diciembre. Obtenido desde http://www. madrimasd.org/revista/revista20/tribuna/tribuna2.asp

Narayanan, V. K. \& Fahey, L. (2005). The relevance of institutional underpinnings of Porter's five forces framework to emerging economies: an epistemological analysis. En Journal of Management Studies, 42( I), enero, pp. 207-223.

Pardo, A. \& Ruiz, M.A. (2002). Guía para el análisis de datos. SPSS I I. Madrid: Mc Graw Hill, Madrid.

Pérez-López, C. (2004). Técnicas de análisis multivariante de datos. Aplicaciones con SPSS. Madrid: Pearson Educación, S.A.

Pérez-López, C. (2005). Métodos estadísticos avanzados con SPSS. Madrid:Thomson.
Schumpeter, J. (1939). Business Cycles: a theoretical, historical and statistical analysis of the capitalist process. Nueva York: Ed. Mc Graw Hill,

Silva, J. (2004). Cuantificando formalmente los coeficientes de innovación de las empresas. Disponible en http://www.rcci. net/globalización/2004/fg421.htm.

Singh, K. \& Mitchell,W. (2005). Growth dynamics: the bidirectional relationship between interfirm collaboration and business sales in entrant and incumbent alliances. En Strategic Management Journal, 26, pp. 497-521.

Teece, D. \& Pisano, G. (1994). The dynamic capabilities of firms: an introduction. En Industrial and Corporate Change, 3(3), pp. 537-556.

Tidd, J. (1993). Tecnhological innovation, organizational linkages and strategic degrees of freedom. En Technology Analysis \& Strategic Management, 5 (3), pp. 273-284.

Tidd, J. (200 I). Innovation management in context: environment, organization and performance. En International Journal of Management Review, 3(3), pp. 169-183.

Tidd, J., \& Bodley, K. (2002). The influence of Project novelty on the new product development process. En, R\&D Management, 32 (2), pp. 127-138.

Tidd, J., Bessant \& K. Pavitt (1997). Managing Innovation. Integrating Technological Gran Bretaña: Market, Wiley.

Visauta, B. \& Martori, J.C. (2003). Análisis estadístico con SPSS para Windows. Volumen II. Estadística multivariante. $2^{\mathrm{a}}$ Ed. Madrid: Ed. Mc Graw Hill.

Zhao, Z., Anand, J. \& Will, M. (2005). A dual networks perspective on inter-organizational transfer of R\&D Capabilities: international joint ventures in the Chinese automotive industry. En Journal of Management Studies, 42(I), enero, pp. 127-160. 